\title{
Perturbative renormalization of staggered fermion operators with stout improvement: Application to the magnetic susceptibility of QCD
}

\author{
G. S. Bali ${ }^{a}$, F. Bruckmann ${ }^{a}$, M. Constantinou ${ }^{b}$, M. Costa $^{* b}$, G. Endrődi ${ }^{a}$, S. D. Katz ${ }^{c}$, \\ H. Panagopoulos ${ }^{b}, \mathbf{A}$. Schäfer ${ }^{a}$ \\ ${ }^{a}$ Institute for Theoretical Physics, Universität Regensburg, D-93040 Regensburg, Germany \\ ${ }^{b}$ Department of Physics, University of Cyprus, Nicosia, CY-1678, Cyprus \\ ${ }^{c}$ Institute for Theoretical Physics, Eötvös University, H-1117, Budapest, Hungary \\ E-mail: gunnar.bali@ur.de, Falk.Bruckmann@physik.uni-regensburg.de, \\ constantinou.martha@ucy.ac.cy, kosta.marios@ucy.ac.cy, \\ Gergely.Endrodi@physik.uni-regensburg.de, katz@bodri.elte.hu, \\ panagopoulos.haris@ucy.ac.cy, \\ Andreas.Schaefer@physik.uni-regensburg.de
}

\begin{abstract}
We calculate the fermion propagator and the quark-antiquark Green's functions for a complete set of ultralocal fermion bilinears, $\mathscr{O}_{\Gamma}[\Gamma$ : scalar $(\mathrm{S})$, pseudoscalar $(\mathrm{P})$, vector $(\mathrm{V})$, axial $(\mathrm{A})$ and tensor (T)], using perturbation theory up to one-loop and to lowest order in the lattice spacing. We employ the staggered action for fermions and the Symanzik Improved action for gluons. From our calculations we determine the renormalization functions for the quark field and for all ultralocal taste-singlet bilinear operators. The novel aspect of our calculations is that the gluon links which appear both in the fermion action and in the definition of the bilinears have been improved by applying a stout smearing procedure up to two times, iteratively. Compared to most other improved formulations of staggered fermions, the above action, as well as the HISQ action, lead to smaller taste violating effects $[1,2,3]$. The renormalization functions are presented in the RI' scheme; the dependence on all stout parameters, as well as on the coupling constant, the number of colors, the lattice spacing, the gauge fixing parameter and the renormalization scale, is shown explicitly.

We apply our results to a nonperturbative study of the magnetic susceptibility of QCD at zero and finite temperature. In particular, we evaluate the "tensor coefficient", $\tau$, which is relevant to the anomalous magnetic moment of the muon.
\end{abstract}

31st International Symposium on Lattice Field Theory LATTICE 2013

July 29 - August 3, 2013

Mainz, Germany

${ }^{*}$ Speaker. 


\section{Introduction}

Renormalization functions (RFs) are necessary ingredients in the prediction of physical probability amplitudes from lattice matrix elements of operators. They relate observables computed on finite lattices to their continuum counterparts in specific renormalization schemes. A set of operators which are of particular interest are fermion bilinears which are widely employed in numerical simulations of Quantum Chromodynamics on the Lattice.

The quark-antiquark Green's functions of "ultralocal" fermion bilinears $\mathscr{O}_{\Gamma}[\Gamma$ : scalar $(\mathrm{S})$, pseudoscalar $(\mathrm{P})$, vector $(\mathrm{V})$, axial $(\mathrm{A})$ and tensor $(\mathrm{T})]$, are calculated perturbatively to one-loop order. We use massive staggered fermions and in the gluon sector we employ the Symanzik Improved gauge action for different sets of values of the Symanzik coefficients. The gluon links which appear both in the fermion action and in the definition of the bilinears have been improved by applying a stout smearing procedure [4] up to two times, iteratively. We implement the RI' renormalization scheme, where the renormalization is determined by comparing the tree-level values of the quark-antiquark Green's functions of the operators with the corresponding values beyond tree-level. Some of the first perturbative results regarding staggered operators and improvements in the action can be found in Refs. [5, 6, 7].

We apply our perturbative results to a study of the response of the QCD vacuum to an external magnetic field, at zero and finite temperature. Magnetic fields probe the QCD vacuum in several ways, by affecting its fundamental properties like chiral symmetry breaking and restoration, the phase diagram, as well as the vacuum polarization. Here we focus on quark condensates, relevant for various phenomenological applications.

The details of our work, along with a longer list of references, can be found in Refs. [8, 9].

\section{Perturbative Renormalization functions}

RFs, for operators and action parameters, relate bare quantities, regularized on the lattice, to their renormalized continuum counterparts:

$$
\psi_{\text {renorm }}=Z_{\mathrm{q}}^{\frac{1}{2}} \psi_{\text {bare }}, \quad m_{\text {renorm }}=Z_{\mathrm{m}} m_{\text {bare }}, \quad \mathscr{O}_{\text {renorm }}^{\Gamma}=Z_{\mathscr{O}_{\Gamma}} \mathscr{O}_{\text {bare }}^{\Gamma} .
$$

We present the RFs, in the RI' scheme, of the quark field $\left(Z_{q}\right)$, the fermion mass $\left(Z_{\mathrm{m}}\right)$ and the taste-singlet quark bilinears: scalar, pseudoscalar, vector, axial, tensor $\left(Z_{\mathrm{S}}, Z_{\mathrm{P}}, Z_{\mathrm{V}}, Z_{\mathrm{A}}, Z_{\mathrm{T}}\right)$.

The one-loop one-particle irreducible (1PI) Feynman diagrams that enter the calculation of the quark-antiquark amputated Green's function (inverse propagator) $S_{1-l o o p}^{-1}$, are illustrated in Fig. 1 . For the algebraic operations involved in evaluating Feynman diagrams, we make use of our symbolic package in Mathematica. A brief description of the procedure for the computation of a Feynman diagram can be found in Ref. [10]. The 1PI Feynman diagrams that enter in the calculation of the two-point Green's functions of the operators, are shown in Fig. 2, and include up to two-gluon vertices extracted from the operator (the cross in the diagrams). The appearance of gluon lines attached to the operators stems from the fact that the definitions of these operators in the staggered formulation contain products of gauge links [5].

We have computed the one-loop inverse fermion propagator, $S^{-1}(p)$, for general values of: the gauge parameter $\alpha$, the stout smearing parameters of the action (for the first and second stout 


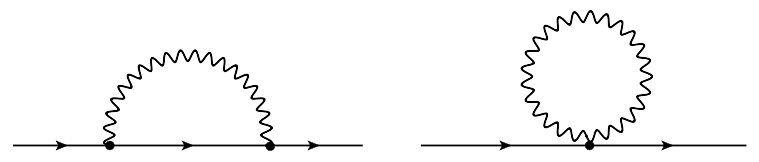

Figure 1: One-loop diagrams contributing to the fermion propagator. Wavy (solid) lines represent gluons (fermions).
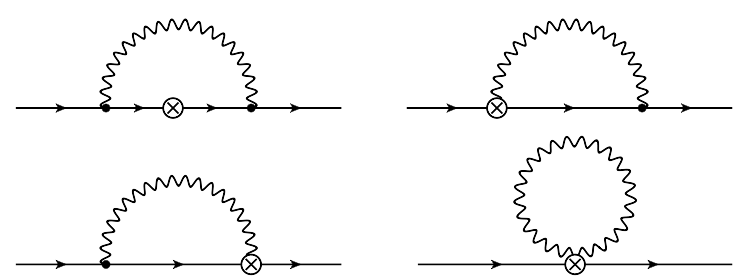

Figure 2: One-loop diagrams contributing to the quark-antiquark Green's functions of the bilinear operators. A wavy (solid) line represents gluons (fermions). A cross denotes an operator insertion.

iteration respectively) $\omega_{A_{1}}, \omega_{A_{2}}$, the Lagrangian mass $m$, the number of colors $N_{c}$ and the external momenta $p_{1}, p_{2}$.

$$
\begin{aligned}
& S_{1-\text { loop }}^{-1}=\frac{i}{a} \sin \left(a p_{1 \rho}\right) \delta\left(p_{1}-p_{2}+\frac{\pi \bar{\rho}}{a}\right) \\
& \quad\left[1+\frac{g^{2} C_{F}}{16 \pi^{2}}\left[\left(e_{1}-\alpha\left(-4.79201+\log \left(a^{2} m^{2}+a^{2} p^{2}\right)+\frac{m^{2}}{p^{2}}-\frac{m^{4}}{p^{4}} \log \left(1+\frac{p^{2}}{m^{2}}\right)\right)\right]\right]\right. \\
& +\delta\left(p_{1}-p_{2}\right) m \\
& \quad\left[1+\frac{g^{2} C_{F}}{16 \pi^{2}}\left[e_{2}+5.79201 \alpha-(3+\alpha)\left(\log \left(a^{2} m^{2}+a^{2} p^{2}\right)+\frac{m^{2}}{p^{2}} \log \left(1+\frac{p^{2}}{m^{2}}\right)\right)\right]\right]
\end{aligned}
$$

where $p_{1}, p_{2}$ : external momenta, $a p_{\rho} \equiv\left(a p_{1 \rho}+\pi / 2\right)_{\bmod \pi}-\pi / 2=\left(a p_{2 \rho}+\pi / 2\right)_{\bmod \pi}-\pi / 2$ and $C_{F} \equiv\left(N_{c}^{2}-1\right) /\left(2 N_{c}\right)$. The quantities $e_{1}, e_{2}$ are numerical coefficients that depend on the stout smearing parameters. We have evaluated $e_{1}, e_{2}$ for several choices of Symanzik Improved gluon actions ; in particular, for the Tree-Level Improved Symanzik gauge action we obtain:

$$
\begin{aligned}
e_{1}= & -7.2136+124.5148\left(\omega_{A_{1}}+\omega_{A_{2}}\right)-518.4332\left(\omega_{A_{1}}^{2}+\omega_{A_{2}}^{2}\right)-2073.7329 \omega_{A_{1}} \omega_{A_{2}} \\
+ & 9435.3458\left(\omega_{A_{1}}^{2} \omega_{A_{2}}+\omega_{A_{1}} \omega_{A_{2}}^{2}\right)-45903.1373 \omega_{A_{1}}^{2} \omega_{A_{2}}^{2}, \\
e_{2}= & 27.1081-264.6953\left(\omega_{A_{1}}+\omega_{A_{2}}\right)+885.2150\left(\omega_{A_{1}}^{2}+\omega_{A_{2}}^{2}\right)+3540.8600 \omega_{A_{1}} \omega_{A_{2}} \\
& -13960.0107\left(\omega_{A_{1}}^{2} \omega_{A_{2}}+\omega_{A_{1}} \omega_{A_{2}}^{2}\right)+60910.8220 \omega_{A_{1}}^{2} \omega_{A_{2}}^{2} .
\end{aligned}
$$

In all numerical results (here and below) the systematic error (coming from an extrapolation to infinite lattice size of our numerical loop-integrals) is smaller than the last digit we present.

We denote the expression in square brackets, in the last line of Eq. (2.2), as $\Sigma_{m}\left(p^{2}, m\right)$; from this we will extract the multiplicative renormalization of the Lagrangian mass, $Z_{\mathrm{m}}$. For bilinear operators $\mathscr{O}_{\Gamma}$, the $\mathrm{RI}^{\prime}$ renormalization scheme consists in imposing that the renormalized forward amputated two-point Green's function, computed in the chiral limit and at a given (large Euclidean) scale $p^{2}=\mu^{2}$, be equal to its tree-level value. The RFs are computed at arbitrary values of the 
renormalization scale $\mu$ [9]. Thus, the $\mathrm{RI}^{\prime}$ conditions for $Z_{\mathrm{q}}, Z_{\mathrm{m}}, Z_{\mathscr{C}_{\Gamma}}$ read:

$$
\begin{aligned}
S_{1-\text { loop }}^{-1}\left(p^{2}=\mu^{2}, m=0\right) & =S_{\text {tree }}^{-1}\left(p^{2}=\mu^{2}, m=0\right) Z_{\mathrm{q}}^{\mathrm{RI}^{\prime}}(\mu) \\
\Sigma_{m}\left(p^{2}=\mu^{2}, m=0\right) & =Z_{\mathrm{m}}^{\mathrm{RI}^{\prime}}(\mu) Z_{\mathrm{q}}^{\mathrm{RI}^{\prime}}(\mu) \\
\Lambda_{\mathscr{O}_{\Gamma}}^{1-\text { loop }}\left(p^{2}=\mu^{2}, m=0\right) & =\Lambda_{\mathscr{O}_{\Gamma}}^{\text {tree }} Z_{\mathrm{q}}^{\mathrm{RI}^{\prime}}(\mu)\left(Z_{\mathscr{O}_{\Gamma}}^{\mathrm{RI}^{\prime}}(\mu)\right)^{-1}, \quad(\Gamma=S, T, P)
\end{aligned}
$$

where $S_{\text {tree }}^{-1}$ is the tree-level result for the inverse propagator, $\Lambda_{\mathscr{O}_{\Gamma}}^{\text {tree }}$ is the tree-level value of the Green's function for $\mathscr{O}_{\Gamma}$ and $\Lambda_{\mathscr{O}_{\Gamma}}^{1-\text { loop }}$ is the bare corresponding Green's function, up to one loop. The one-loop Green's functions for $\mathscr{O}_{V}$ and $\mathscr{O}_{A}$ contain two Lorentz structures each:

$$
\Lambda_{\mathscr{O}_{V}}^{1-\text { loop }}=\gamma_{\mu} \Sigma_{V}^{(1)}(p)+\frac{p_{\mu} \not p}{p^{2}} \Sigma_{V}^{(2)}(p), \Lambda_{\mathscr{O}_{A}}^{1-\text { loop }}=\gamma_{5} \gamma_{\mu} \Sigma_{A}^{(1)}(p)+\gamma_{5} \frac{p_{\mu} \not p}{p^{2}} \Sigma_{A}^{(2)}(p),
$$

$\left(\Sigma_{V, A}^{(2)}(p)=\mathscr{O}\left(g^{2}\right)\right)$. The presence of $\Sigma_{V}^{(2)}$ and $\Sigma_{A}^{(2)}$ makes a prescription such as Eq. (2.7) inapplicable in those cases. Instead we apply renormalization conditions only on $\Sigma_{V, A}^{(1)}$ :

$$
\gamma_{\mu} \Sigma_{V}^{(1)}\left(p^{2}=\mu^{2}, m=0\right)=\Lambda_{\mathscr{O}_{V}}^{\text {tree }} Z_{\mathrm{q}}^{\mathrm{RI}^{\prime}}(\mu)\left(Z_{\mathscr{O}_{V}}^{\mathrm{RI}^{\prime}}(\mu)\right)^{-1}
$$

(and similarly for $\mathscr{O}_{A}$ ). We have also applied two stout-smearing steps to the links in the definition of bilinears, with stout parameters $\omega_{\mathscr{O}_{1}}$ and $\omega_{\mathscr{O}_{2}}$; for general applicability all stout parameters $\left(\omega_{A_{1}}, \omega_{A_{2}}, \omega_{\mathscr{O}_{1}}, \omega_{\mathscr{O}_{2}}\right)$ have been kept distinct.

Our results for $Z_{\mathrm{q}}$ and $Z_{\mathscr{O}_{\Gamma}}$ are presented below; we note that $Z_{\mathrm{m}}$ and $Z_{\mathrm{S}}$ turn out to be related by $Z_{\mathrm{m}}=Z_{\mathrm{S}}^{-1}$, as was expected.

$$
\begin{aligned}
& Z_{q}^{\mathrm{RI}}=1+\frac{g^{2} C_{F}}{16 \pi^{2}}\left[-7.2136+4.7920 \alpha+124.5149\left(\omega_{A_{1}}+\omega_{A_{2}}\right)-518.4332\left(\omega_{A_{1}}{ }^{2}+\omega_{A_{2}}{ }^{2}\right)\right. \\
& -2073.7329 \omega_{A_{1}} \omega_{A_{2}}+9435.3459\left(\omega_{A_{1}}{ }^{2} \omega_{A_{2}}+\omega_{A_{1}} \omega_{A_{2}}{ }^{2}\right) \\
& \left.-45903.1373 \omega_{A_{1}}{ }^{2} \omega_{A_{2}}{ }^{2}+\alpha \log \left(a^{2} \mu^{2}\right)\right] \\
& Z_{S}^{\mathrm{RI}^{\prime}}=1+\frac{g^{2} C_{F}}{16 \pi^{2}}\left[-34.3217-\alpha+389.2102\left(\omega_{A_{1}}+\omega_{A_{2}}\right)-1403.6482\left(\omega_{A_{1}}{ }^{2}+\omega_{A_{2}}{ }^{2}\right)\right. \\
& -5614.5930 \omega_{A_{1}} \omega_{A_{2}}+23395.3566\left(\omega_{A_{1}}^{2} \omega_{A_{2}}+\omega_{A_{1}} \omega_{A_{2}}{ }^{2}\right) \\
& \left.-106813.9602 \omega_{A_{1}}^{2} \omega_{A_{2}}^{2}+3 \log \left(a^{2} \mu^{2}\right)\right]=\left(Z_{m}^{\mathrm{RI}^{\prime}}\right)^{-1} \\
& Z_{P}^{\mathrm{RI}^{\prime}}=1+\frac{g^{2} C_{F}}{16 \pi^{2}}\left[25.7425-\alpha+119.0620\left(\omega_{A_{1}}+\omega_{A_{2}}\right)-428.1202\left(\omega_{\mathscr{O}_{1}}+\omega_{\mathscr{O}_{2}}\right)\right. \\
& -518.5414\left(\omega_{A_{1}}{ }^{2}+\omega_{A_{2}}{ }^{2}\right) \\
& +1667.0015\left(\omega_{\mathscr{O}_{1}}{ }^{2}+\omega_{\mathscr{O}_{2}}{ }^{2}\right)-2042.2891 \omega_{A_{1}} \omega_{A_{2}}+6699.8826 \omega_{\mathscr{O}_{1}} \omega_{\mathscr{O}_{2}} \\
& +31.8765\left(\omega_{A_{1}}+\omega_{A_{2}}\right)\left(\omega_{\mathscr{O}_{1}}+\omega_{\mathscr{O}_{2}}\right)+9435.3986\left(\omega_{A_{1}}{ }^{2} \omega_{A_{2}}+\omega_{A_{1}} \omega_{A_{2}}{ }^{2}\right) \\
& -29653.9826\left(\omega_{\mathscr{O}_{1}}{ }^{2} \omega_{\mathscr{O}_{2}}+\omega_{\mathscr{O}_{1}} \omega_{\mathscr{O}_{2}}{ }^{2}\right)-210.2738\left(\omega_{A_{1}}+\omega_{A_{2}}\right) \omega_{\mathscr{O}_{1}} \omega_{\mathscr{O}_{2}} \\
& -210.2738 \omega_{A_{1}} \omega_{A_{2}}\left(\omega_{\mathscr{O}_{1}}+\omega_{\mathscr{O}_{2}}\right)-44803.9568 \omega_{A_{1}}{ }^{2} \omega_{A_{2}}{ }^{2} \\
& +143482.2565 \omega_{\mathscr{O}_{1}}^{2} \omega_{\mathscr{O}_{2}}^{2}+1657.7660 \omega_{A_{1}} \omega_{A_{2}} \omega_{\mathscr{O}_{1}} \omega_{\mathscr{O}_{2}} \\
& \left.+3 \log \left(a^{2} \mu^{2}\right)\right]
\end{aligned}
$$




$$
\begin{aligned}
& Z_{V}^{\mathrm{RI}^{\prime}}=1+\frac{g^{2} C_{F}}{16 \pi^{2}}\left[\quad 86.7568\left[\left(\omega_{A_{1}}+\omega_{A_{2}}\right)-\left(\omega_{\mathscr{O}_{1}}+\omega_{\mathscr{O}_{2}}\right)\right]-337.3834\left[\left(\omega_{A_{1}}{ }^{2}+\omega_{A_{2}}{ }^{2}\right)\right.\right. \\
& \left.-\left(\omega_{\mathscr{O}_{1}}^{2}+\omega_{\mathscr{O}_{2}}^{2}\right)\right]-1349.5337\left(\omega_{A_{1}} \omega_{A_{2}}-\omega_{\mathscr{O}_{1}} \omega_{\mathscr{O}_{2}}\right) \\
& +5950.8059\left[\left(\omega_{A_{1}}{ }^{2} \omega_{A_{2}}+\omega_{A_{1}} \omega_{A_{2}}{ }^{2}\right)-\left(\omega_{\mathscr{O}_{1}}{ }^{2} \omega_{\mathscr{O}_{2}}+\omega_{\mathscr{O}_{1}} \omega_{\mathscr{O}_{2}}{ }^{2}\right)\right] \\
& \left.-28627.2520\left(\omega_{A_{1}}^{2}{\omega_{A_{2}}}^{2}-\omega_{\mathscr{O}_{1}}{ }^{2}{\omega_{\mathscr{O}_{2}}}^{2}\right)\right] \\
& Z_{A}^{\mathrm{RI}}=1+\frac{g^{2} C_{F}}{16 \pi^{2}}\left[17.0363+117.5844\left(\omega_{A_{1}}+\omega_{A_{2}}\right)-314.3549\left(\omega_{\mathscr{O}_{1}}+\omega_{\mathscr{O}_{2}}\right)\right. \\
& -518.4189\left(\omega_{A_{1}}^{2}+\omega_{A_{2}}^{2}\right) \\
& +1223.7950\left(\omega_{\mathscr{O}_{1}}{ }^{2}+\omega_{\mathscr{O}_{2}}{ }^{2}\right)-2041.7996 \omega_{A_{1}} \omega_{A_{2}}+4927.0558 \omega_{\mathscr{O}_{1}} \omega_{\mathscr{O}_{2}} \\
& +31.8758\left(\omega_{A_{1}}+\omega_{A_{2}}\right)\left(\omega_{\mathscr{O}_{1}}+\omega_{\mathscr{O}_{2}}\right)+9559.9779\left({\omega_{A_{1}}}^{2} \omega_{A_{2}}+\omega_{A_{1}} \omega_{A_{2}}{ }^{2}\right) \\
& -21823.5425\left(\omega_{\mathscr{O}_{1}}{ }^{2} \omega_{\mathscr{O}_{2}}+\omega_{\mathscr{O}_{1}} \omega_{\mathscr{O}_{2}}{ }^{2}\right)-210.2735\left(\omega_{A_{1}}+\omega_{A_{2}}\right) \omega_{\mathscr{O}_{1}} \omega_{\mathscr{O}_{2}}
\end{aligned}
$$

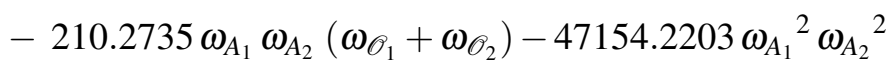

$$
\begin{aligned}
& \left.+105753.7547 \omega_{\mathscr{O}_{1}}^{2} \omega_{\mathscr{O}_{2}}{ }^{2}+1396.9376 \omega_{A_{1}} \omega_{A_{2}} \omega_{\mathscr{O}_{1}} \omega_{\mathscr{O}_{2}}\right]
\end{aligned}
$$

$$
\begin{aligned}
Z_{T}^{\mathrm{RI}}=1+\frac{g^{2} C_{F}}{16 \pi^{2}}[ & 8.8834+\alpha+116.5787\left(\omega_{A_{1}}+\omega_{A_{2}}\right)-200.5879\left(\omega_{\mathscr{O}_{1}}+\omega_{\mathscr{O}_{2}}\right) \\
& -531.7591\left(\omega_{A_{1}}{ }^{2}+\omega_{A_{2}}{ }^{2}\right)+780.5904\left(\omega_{\mathscr{O}_{1}}{ }^{2}+\omega_{\mathscr{O}_{2}}{ }^{2}\right) \\
& -2095.1622 \omega_{A_{1}} \omega_{A_{2}}+3154.2357 \omega_{\mathscr{O}_{1}} \omega_{\mathscr{O}_{2}}+31.8743\left(\omega_{A_{1}}+\omega_{A_{2}}\right)\left(\omega_{\mathscr{O}_{1}}+\omega_{\mathscr{O}_{2}}\right) \\
& +9877.2330\left(\omega_{A_{1}}{ }^{2} \omega_{A_{2}}+\omega_{A_{1}} \omega_{A_{2}}{ }^{2}\right)-13993.1045\left(\omega_{\mathscr{O}_{1}}{ }^{2} \omega_{\mathscr{O}_{2}}+\omega_{\mathscr{O}_{1}} \omega_{\mathscr{O}_{2}}{ }^{2}\right) \\
& -284.0013\left[\left(\omega_{A_{1}}+\omega_{A_{2}}\right) \omega_{\mathscr{O}_{1}} \omega_{\mathscr{O}_{2}}+\omega_{A_{1}} \omega_{A_{2}}\left(\omega_{\mathscr{O}_{1}}+\omega_{\mathscr{O}_{2}}\right)\right] \\
& -48519.2862 \omega_{A_{1}}{ }^{2} \omega_{A_{2}}{ }^{2}+68237.1178 \omega_{\mathscr{O}_{1}}{ }^{2} \omega_{\mathscr{O}_{2}}{ }^{2} \\
& \left.+2709.4942 \omega_{A_{1}} \omega_{A_{2}} \omega_{\mathscr{O}_{1}} \omega_{\mathscr{O}_{2}}-\log \left(a^{2} \mu^{2}\right)\right] .
\end{aligned}
$$

We also note in passing that in the absence of stout smearing $\left(\omega_{A_{i}}=\omega_{\mathscr{C}_{i}}=0\right) Z_{\mathrm{V}}^{\mathrm{RI}^{\prime}}=1$, as is well known from current conservation. In addition, Eq. (2.13) shows that non-renormalization of $\mathscr{O}_{V}$ applies also when $\omega_{A_{i}}=\omega_{\mathscr{O}_{i}}$; this follows from the fact that the stout link version of $\mathscr{O}_{V}$ mimics the fermion action, and thus current conservation applies equally well in this case.

To obtain $Z_{\mathscr{O}_{\Gamma}}$ in the $\overline{\mathrm{MS}}$ scheme, we apply conversion factors, $C_{\mathscr{C}_{\Gamma}}$, which have been computed in dimensional regularization [11].

$$
Z_{\mathscr{O}_{\Gamma}}^{\overline{\mathrm{MS}}}=C_{\mathscr{O}_{\Gamma}} Z_{\mathscr{O}_{\Gamma}}^{\mathrm{RI}}
$$

These conversion factors do not depend on the regularization scheme. Furthermore, they refer to the Naive Dimensional Regularization (NDR) of the $\overline{\mathrm{MS}}$ scheme, in which $C_{P}=C_{S}$ and $C_{A}=C_{V}$. From Eq. (2.16) one obtains:

$$
Z_{\mathrm{q}}^{\overline{\mathrm{MS}}}=Z_{\mathrm{q}}^{\mathrm{RI}}-\frac{g^{2} C_{F}}{16 \pi^{2}} \alpha, \quad Z_{\mathrm{S}, \mathrm{P}}^{\overline{\mathrm{MS}}}=Z_{\mathrm{S}, \mathrm{P}}^{\mathrm{RI}}+\frac{g^{2} C_{F}}{16 \pi^{2}}(4+\alpha), \quad Z_{\mathrm{V}, \mathrm{A}}^{\overline{\mathrm{MS}}}=Z_{\mathrm{V}, \mathrm{A}}^{\mathrm{RI}}, \quad Z_{\mathrm{T}}^{\overline{\mathrm{MS}}}=Z_{\mathrm{T}}^{\mathrm{RI}}-\frac{g^{2} C_{F}}{16 \pi^{2}} \alpha .
$$




\section{Simulation setup}

We study the effect of an external magnetic field, B, on the expectation value of the tensor polarization [8]. The leading order of the expectation value of the tensor polarization operator $\bar{\psi}_{f} \sigma_{\mu \nu} \psi_{f}$, is proportional to the field strength and thus can be written as:

$$
\left\langle\bar{\psi}_{f} \sigma_{x y} \psi_{f}\right\rangle=q_{f} B \cdot\left\langle\bar{\psi}_{f} \psi_{f}\right\rangle \cdot \chi_{f} \equiv q_{f} B \cdot \tau_{f},
$$

where $\left\langle\bar{\psi}_{f} \psi_{f}\right\rangle$ is the quark condensate and $\chi_{f}$ is the "magnetic susceptibility" of the condensate. We define the "tensor coefficient" $\tau_{f}$ as the product of the condensate and the magnetic susceptibility of the condensate.

The bare observable can be written as:

$$
\left\langle\bar{\psi}_{f} \psi_{f}\right\rangle(B, T)=\frac{1}{Z_{\mathrm{S}}}\left\langle\bar{\psi}_{f} \psi_{f}\right\rangle_{\text {renorm }}(B, T)+\zeta_{S} m_{f} / a^{2}+\ldots,
$$

where the divergences in $\left\langle\bar{\psi}_{f} \psi_{f}\right\rangle$ depend neither on the temperature nor on the external field. Therefore, in mass-independent renormalization schemes, $\zeta_{S}$ and $Z_{S}$ are just functions of the gauge coupling. Similarly,

$$
\left\langle\bar{\psi}_{f} \sigma_{\mu \nu} \psi_{f}\right\rangle(B, T)=\frac{1}{Z_{\mathrm{T}}}\left\langle\bar{\psi}_{f} \sigma_{x y} \psi_{f}\right\rangle_{\text {renorm }}(B, T)+\zeta_{T} q_{f} B m_{f} \log \left(m_{f}^{2} a^{2}\right)+\ldots,
$$

where $\zeta_{T}$ is the coefficient of the divergent logarithm. Both $Z_{T}$ and $\zeta_{T}$ are independent of $T$ and $B$ (and $m_{f}$, in mass-independent schemes). In Eq. (3.3) the ellipses denote finite terms. In the free theory we calculate $\zeta_{T}(g=0)=3 /\left(4 \pi^{2}\right)$. We use the perturbative $Z_{S}$ and $Z_{T}$ in $\overline{\mathrm{MS}}$ scheme with parameters $\omega_{\mathscr{O}_{i}}=\omega_{A_{i}}=0.15$. We also notice that the operator $1-m_{f} \partial / \partial m_{f}$ eliminates the logarithmic divergence and thus can be used to define an observable with a finite continuum limit,

$$
\tau_{f}^{\mathrm{renorm}} \equiv \tau_{f}^{r}=\left(1-m_{f} \frac{\partial}{\partial m_{f}}\right) \tau_{f} \cdot Z_{\mathrm{T}} \equiv \tau_{f} Z_{\mathrm{T}}-\tau_{f}^{\mathrm{div}}
$$

where $\tau_{f}^{\mathrm{div}}$ is independent of the temperature. We measure $Z_{T} \cdot \tau_{f}$, with the value of $m_{s}$ fixed to its physical value and we tune only $m_{u d}\left(R \equiv m_{u d} / m_{u d}^{\text {phys }}\right)$ for $T=0$ and $0.5<R<28.15$. We consider the following fit function for $Z_{T} \cdot \tau_{f}$ :

$$
c_{f 0}+c_{f 1} R+c_{f 2} R \log \left(R^{2} a^{2}\right), \quad c_{f i}=c_{f i}^{(0)}+c_{f i}^{(1)} a^{2}
$$

Appling the operator $1-m_{f} \partial_{m_{f}}=1-R \partial_{R}$ we find the value of $\tau_{f}^{r}$ at $T=0$. We translate these results to the magnetic susceptibility $\chi_{f}$ of Eq. (3.1) using the value of the quark condensate. The zero-temperature magnetic susceptibilities (in the $\overline{\mathrm{MS}}$ scheme at scale $\mu=2 \mathrm{GeV}$ ) are:

$$
\chi_{u}=-(2.08 \pm 0.08) \mathrm{GeV}^{-2}, \quad \chi_{d}=-(2.02 \pm 0.09) \mathrm{GeV}^{-2}, \quad \chi_{s}=-(3.4 \pm 1.4) \mathrm{GeV}^{-2} .
$$

We observe that the dependence of the condensate on $B$ varies strongly with the temperature in the transition region. More details on the simulation setup and nonperturbative results can be found in Refs. [8, 12]. 


\section{Conclusion}

We have determined the renormalization functions of taste-singlet bilinear fermion operators. The novelty in our perturbative calculations is the stout smearing of the links that we apply in both the fermion action and in the bilinear operators. More precisely, we use two steps of stout smearing with distinct parameters. To make our results as general as possible we also distinguish between the stout parameters appearing in the fermion action and in the bilinears.

In Ref. [8, 12], we measure the magnetic susceptibilities $\chi_{f}$ at zero temperature for the up, down and strange quarks in the $\overline{\mathrm{MS}}$ scheme at a renormalization scale of $2 \mathrm{GeV}$. The magnetic susceptibilities at $T=0$ are negative, indicating the spin-diamagnetic nature of the QCD vacuum. We also find that the polarization changes smoothly with temperature in the confinement phase and then drastically reduces around the transition region.

Acknowledgements: Our work was supported by the Cyprus Research Promotion Founda-

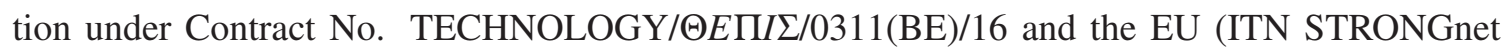
238353).

\section{References}

[1] Y. Aoki, Z. Fodor, S.D. Katz, and K. Szabo, J. High Energy Phys. 01 (2006) 089 [arXiv:hep-lat/0510084].

[2] S. Borsanyi, Z. Fodor, S. D. Katz, S. Krieg, C. Ratti, et al., J. Phys. G38 (2011) 124060 [arXiv:1109.5030].

[3] A. Bazavov, T. Bhattacharya, M. Cheng, C. DeTar, H. Ding et al., Phys. Rev. D85 (2012) 054503 [arXiv:1111.1710].

[4] C. Morningstar and M. Peardon, Phys. Rev. D69 (2004) 054501 [arXiv: hep-lat/ 0311018 ].

[5] A. Patel and S. Sharpe, Nucl. Phys. B395 (1993) 701-732 [arXiv: hep-lat/9210039].

[6] N. Ishizuka and Y. Shizawa, Phys. Rev. D49 (1994) 3519-3539 [arXiv : hep-lat/930 8008 ].

[7] W. Lee and S. R. Sharpe, Phys. Rev. D66 (2002) 114501 [arXiv: hep-lat/0208018].

[8] G. S. Bali, F. Bruckmann, M. Constantinou, M. Costa, G. Endrődi, et al., Phys. Rev. D86 (2012) 094512 [arXiv:1209.6015].

[9] M. Constantinou, M. Costa and H. Panagopoulos, Phys. Rev. D88 (2013) 034504 [arXiv:1305.1870].

[10] M. Constantinou, V. Lubicz, H. Panagopoulos and F. Stylianou, JHEP 0910 (2009) 064 [arXiv:0907.0381].

[11] J. A. Gracey, Nucl. Phys. B662 (2003) 247-278 [arXiv: hep-ph/0304113].

[12] G. S. Bali, F. Bruckmann, M. Constantinou, M. Costa, G. Endrődi, et al., Pos Confinement X (2012) $198[\operatorname{arXiv}: 1301.5826]$. 